\title{
Coherent Control of LO-Phonon Dynamics in Opaque Semiconductors by Femtosecond Laser Pulses.
}

\author{
T. Dekorsy, W. KüTT, T. Pfeifer and H. KurZ \\ Institut für Halbleitertechnik, Rheinisch Westfälische Technische Hochschule Aachen \\ Sommerfeldstr. 24, D-5100 Aachen, Germany
}

\begin{abstract}
Coherent longitudinal optical phonons are generated via ultrafast screening of GaAs surface-space-charge fields by free carriers excited with femtosecond laser pulses. The coherent phonons are observed in a pump probe experiment through anisotropic changes in the refractive index. Since the electro-optic observation of phonons in the time domain allows the determination of both the amplitude and the phase of coherent lattice vibrations, we are able to superpose two coherently excited phonon modes in a dual excitation experiment. By carefully adjusting the relative phase, we are able to accurately control the amplitude of the coherent vibrations from resonant enhancement to total extinction. The experiment demonstrates the possibility of a coherent control of the lattice dynamics in solid-state materials at room temperature.
\end{abstract}

Introduction. - The observation of coherently excited phonons in the time domain has become possible by the development of ultrashort laser pulses with pulse duration shorter than the oscillation period. Optically stimulated coherent vibrations have been observed in dyes, molecular crystals and condensed media, revealing physical insight into the excitation and dephasing mechanisms [1-9]. In opaque semiconductors like GaAs, the generation of coherent LO phonons with femotosecond laser pulses is based on the screening of the surface-space-charge field via ultrafast photocurrents $[10,11]$. This generation mechanism significantly differs from other generation mechanisms as displacive excitation of coherent phonons (DECP) [12] or impulsive stimulated Raman scattering (ISRS) [13].

The field screening at GaAs surfaces results from free-carrier transport initiated by above-band-gap pulsed optical excitation. The decrease of the surface field as well as the oscillation of the macroscopic polarization associated with the coherent LO phonons are detected via the linear electro-optic effect in a reflective geometry. In contrast to c.w. Raman scattering experiments, both amplitude and phase of the lattice vibration can be detected. This allows optical experiments for the observation of the superposition of coherent phonons. A second pump pulse - with a variable time delay with respect to the firstgenerates a second burst of coherent phonons within the surface-space-charge field, which superpose constructively or destructively with the phonons already present, depending on 
the relative phase. Such an experiment has strong analogy with ISRS experiments by Weiner and co-workers [14], where a periodic laser pulse train is used for the resonant enhancement of coherent vibrations in a molecular crystal at $5 \mathrm{~K}$. There, the vibrational amplitudes are mode selectively amplified when the frequency spacing of the pulse train matches the oscillation frequency (approximately $2.4 \mathrm{THz}$ ). Although the present dual-excitation experiment can be regarded as a pulse train experiment, where the pulse train consists only of two successive pulses, it differs in several aspects from the ISRS experiments on molecular crystals. The investigated material is a non-transparent semiconductor, the experiment is performed at room temperature and the involved oscillation period of $8.8 \mathrm{THz}$ is significantly higher. In addition the excitation mechanism is incoherent carrier transport in our case compared to a coherent process in ISRS. Both experiments demonstrate how the collective atomic motion can be controlled accurately by femtosecond laser pulses.

Generation and detection of coherent phonons. - The generation of coherent LO phonons in GaAs space-charge fields is based on a coupling of the lattice displacement to a macroscopic electric field [11,9]. The macroscopic electric field is set up by the Fermi-level pinning at the bare semiconductor surface due to charged surface states [15]. This effect results in a band bending towards the surface. The amplitude and screening length of the associated electric field perpendicular to the surface mainly depends on the doping density of the sample and the magnitude of the band bending [16]. It can be easily controlled via a Schottky contact and an externally applied voltage.

Optical excitation of free carriers with an ultrashort laser pulse from a colliding-pulse mode-locked $(\mathrm{CPM})$ ring dye laser $\left(\tau_{\mathrm{FWHM}}=50 \mathrm{fs}, E_{\text {laser }}=2 \mathrm{eV}\right.$ ) results in a rapid screening of the surface field via free-carrier transport $[11,17]$. This screening occurs on the time scale of the pulse duration, which is shorter than one LO-phonon period $\nu_{\text {LO }}^{-1} \approx 114 \mathrm{fs}$ at $k=0$. The ionic displacements in the polar semiconductor couple to the macroscopic field resulting in a coherent excitation of lattice vibrations within the surface-space-charge field.

We use reflective electro-optic sampling (REOS) for the detection of the ultrafast surface field changes on (100)-oriented GaAs [18]. The electric field causes anisotropic changes in the refractive index due to the linear electro-optic effect [19]. We use the symmetry of the effect on (100)-oriented GaAs, which causes changes in the refractive index along the [0 $\overline{1} 1]$ and $[0 \overline{11}]$ directions due to an electric field along the [100] directions. Therefore the probe pulse polarization is oriented along the [001] axis of the sample. The reflected probe beam is split into two orthogonal parts along the [01ㅣ and [0 $\overline{11}]$ directions. The resulting difference signal is sensitive to longitudinal field changes only,

$$
\Delta R(t)=R_{[0 \overline{1} 1]}(t)-R_{[0 \overline{11}]}(t) \sim n_{0}^{3} r_{41} \Delta E(t),
$$

where $n_{0}$ is the isotropic refractive index, $r_{41}$ is the electro-optic coefficient of GaAs $\left(1.6 \cdot 10^{-10} \mathrm{~cm} / \mathrm{V}[19]\right)$ and $E$ is the longitudinal surface field. We use a fast-signal averaging technique by accomplishing the time delay via a retroreflector mounted onto a shaker. The difference signal is amplified and directly recorded in a high-performance computer unit (4 $\mathrm{MHz} \mathrm{A} / \mathrm{D}$-converter) as a function of the time-calibrated shaker displacement. This technique provides a high signal resolution for the change in the reflectivity up to a few $10^{-7}[20]$, corresponding to a sensitivity for field changes of approximately $100 \mathrm{~V} / \mathrm{cm}$.

The coherently generated phonons are observed via periodic modulations of the anisotropic refractive index. This macroscopic electric-field oscillation set up by the coherent lattice motion is superposed to the field screening contribution. For the analysis of the coherent phonons only the screening contribution is numerically subtracted. 
The experimentally observed screening dynamics is well reproduced by numerical solutions of a drift diffusion model [17]. The calculation of the electro-optic response allows a quantitative determination of the observed signal amplitude and dynamics. The calculation confirms an observed saturation of the REOS signals at excitation densities $>10^{18} \mathrm{~cm}^{-3}$, due to the complete screening of the surface-space-charge field, which also means that the generation mechanism for further coherent-phonon generation is saturated.

Superposition of coherent LO phonons. - For the dual-excitation experiment we use an $n$-type GaAs sample $\left(N_{\mathrm{D}}=(3 \div 4) \cdot 10^{17} \mathrm{~cm}^{-3}\right)$ with a transparent indium-tin-oxide (ITO) Schottky contact. The rear of the sample consists of an Ohmic contact, so an adjustment of the built-in field via an externally applied bias voltage is possible.

To demonstrate coherent-LO-phonon superposition we use the second output beam of the CPM laser as a second pump pulse. This beam can be used, because we do not observe a jitter between the two counterpropagating CPM pulses at the time scale of the phonon period. There exist two conditions for the superposition of coherent phonons. The first condition concerns the phonon dephasing time, which presents no problem, since the dephasing time is $T_{2} \approx 4 \mathrm{ps}$ at room temperature [21] and sufficient low excitation levels. The second condition concerns the saturation of the generation process, which is achieved at excitation densities above $10^{18} \mathrm{~cm}^{-3}$, is more difficult to meet. In order to achieve total extinction of the two coherent-phonon modes, the driving force for both phonon modes has to be adjusted to equal magnitude.

The intensities of the two successive pump pulses are adjusted in a way that the second pulse has about $15 \%$ more intensity than the first. This is necessary because screening is a sublinear function of carrier density [17]. The first pump pulse generates a carrier density of approximately $4 \cdot 10^{17} \mathrm{~cm}^{-3}$. The density of the primary excitation should not exceed densities higher than $10^{18} \mathrm{~cm}^{-3}$, because then the plasma frequency comes into resonance with the LO-phonon frequency. This leads to the appearance of an additional TO mode (screened LO phonon), which is observed as a mode beating between LO and TO frequency in the time domain [22]. Additionally, the dephasing time decreases at these carrier densities. At excitation below $10^{17} \mathrm{~cm}^{-3}$, the initial fast screening is insufficient to generate coherent phonons. Thus, there exists a narrow density range of $10^{17} \mathrm{~cm}^{-3}<N<10^{18} \mathrm{~cm}^{-3}$ in which a phonon superposition experiment can be properly performed.

Figure 1 shows the REOS signals for dual excitation with a time delay $\Delta t_{12}$ of approximately $600 \mathrm{fs}$ between the two pump pulses. The single-excitation signals are also

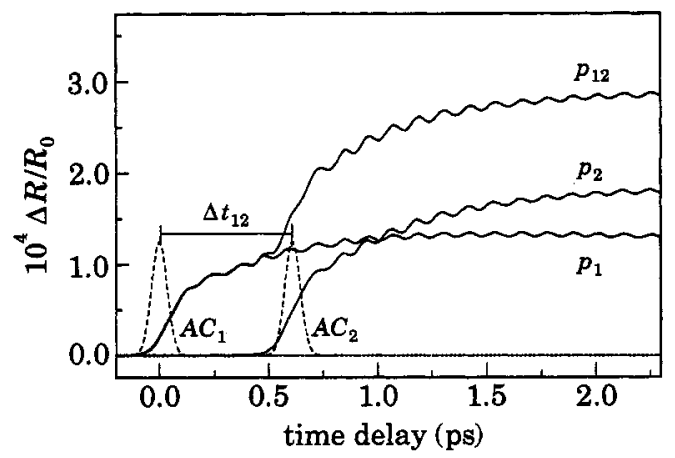

Fig. 1. - Two-pump-pulse experiment at the ITO $/ n$-type GaAs sample at $2.1 \mathrm{~V}$ reverse bias. $p_{1}$ and $p_{2}$ denote the signals from single excitation and $A C_{1}$ and $A C_{2}$ denote their autocorrelation functions (dashed lines). $p_{12}$ is the signal for dual excitation. 


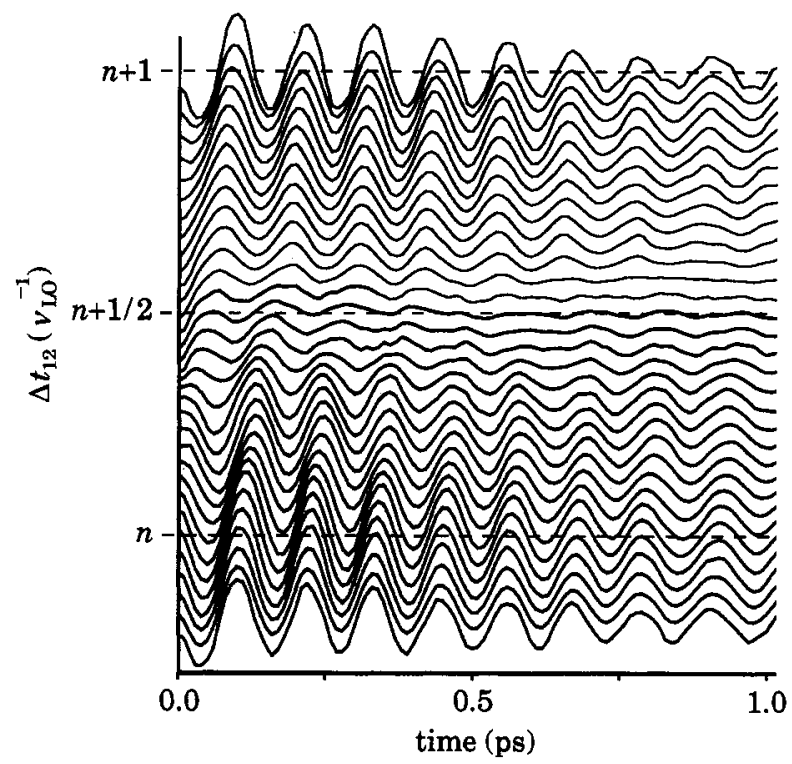

Fig. 2. - Superposition of coherent LO phonons. $\Delta t_{12}$ is the time delay of the two pump pulses in units of the LO-phonon period $v_{\mathrm{LO}}^{-1} \approx 114 \mathrm{fs}$, while the time axis gives the delay of the probe pulse with respect to the first pump pulse.

shown (curves labelled $p_{1}$ and $p_{2}$ ) and reveal a fast initial screening during the excitation pulse followed by a slower rise over a few picoseconds. This screening dynamies results from the temporal evolution of spatial carrier distributions [17]. The REOS signals are clearly modulated by coherently generated LO phonons with a frequency of $8.75 \mathrm{THz}$.

The reverse bias applied to the ITO $/ n$-GaAs sample is $2.1 \mathrm{~V}$. The associated maximum electric field at the surface is about $500 \mathrm{kV} / \mathrm{cm}$ and the screening length $90 \mathrm{~nm}$, both calculated from the Schottky model[16]. At this voltage we achieve equal screening amplitude and dynamics for the second pulse in the presence of the first, which means that the driving forces for the coherent phonons are approximately equal. The dephasing of the first mode has to be taken into account, which results in a decrease of the oscillation amplitude $Q$, e.g. $Q(t=600 \mathrm{fs}) \approx 0.86 Q(t=0)$. The sublinearity of the screening as a function of carrier density may be proved by subtracting the curve labelled $p_{1}$ (only first pulse present) from the one labelled $p_{12}$ (both pump pulses), which yields a signal lower than the signal when only the second pulse is present $\left(p_{2}\right)$. At these experimental conditions we tune the time delay $\Delta t_{12}$ between the two pump pulses over one phonon period $\nu_{\mathrm{LO}}^{-1}$. Figure 2 depicts the extracted oscillatory part of this experiment. The time delay $\Delta t_{12}$ covers one full vibrational period. Destructive superposition is observed at $\Delta t_{12}=(n+1 / 2) \nu_{\mathrm{LO}}^{-1}$, while the coherent-phonon amplitude is resonantly enhanced when the time delay is an integer times the phonon period. The oscillation amplitude at the point of constructive interference is twice the amplitude resulting from a single pump pulse.

The limits we found to be relevant in our experiment are comparable to the limitation in multiple-pulse ISRS experiments [14]. There, one limitation is the pulse width and the pulse repetition period in the pulse train, which presently limits the resolved and enhanced oscillations to about $6 \mathrm{THz}$ [14]. In our experiment only the pulse width is relevant as far as the excitation and detection of coherent phonons are concerned, which presently limits our temporal resolution to approximately $12 \mathrm{THz}$. In the ISRS experiments a resonant mode 
selective enhancement is possible, when the dephasing time $T_{2}$ is larger than the length of the pulse train, which is quite the same condition for the time delay of the two pump pulses we used. In the dual-excitation experiment, however, it is possible to make the coherent atomic motion unambiguously go and stop for a well-defined time.

Conclusion. - We demonstrated the superposition of coherently generated LO phonons in GaAs by applying femtosecond pump probe techniques. Coherent LO phonons are generated via the ultrafast screening of surface-space-charge fields due to the transport of optically excited free carriers. Two successive pump pulses are used for the independent generation of coherent phonons. By adjusting the time delay between the two pulses, we can control the amplitude of the superposed coherent modes accurately via their relative phase. The limits for the observation of coherent-phonon superposition have been fulfilled, so that even total extinction of the coherently generated phonons has become possible.

This method of superposing coherent phonons and accurately controlling the amplitude of the vibrational mode between resonant enhancement and extinction opens the way for some new spectroscopic experiments. For example, this experiment can be performed at low temperatures, well below the thermal activation energy for LO phonons. Dual excitation at low carrier excess energies (< LO-phonon energy) allows to switch coherent LO phonons on and off for a short period and observe the response in carrier dynamics on the short phonon burst. Furthermore the possibility of switching on coherent LO phonons for a well-defined time, say one-half phonon period of approximately $57 \mathrm{fs}$ with a sufficient short laser pulse, allows to generate coherent acoustic phonons by the decaying LO phonons $\left(\nu_{L O}>\nu_{L A}\right)$. The generation of coherent acoustic modes is of particular interest for experiments involving coherent non-equilibrium phonons, as has been recently proposed [23].

We would like to thank H. J. BAKKER for fruitful discussions and B. GöRIG (Humboldt University Berlin) for supplying the ITO-GaAs sample. This work was entirely supported by the "Deutsche Forschungsgemeinschaft".

\section{REFERENCES}

[1] Laubereau A. and Kaiser W., Rev. Mod. Phys., 50 (1978) 607.

[2] DeSilvestri S., Fujimoto J. G., Gamble E. B., Williams L. R. and Nelson K. A., Chem. Phys. Lett., 116 (1985) 146.

[3] Rosker M. J., Wise F. W. and Tang C. L., Phys. Rev. Lett., 57 (1986) 321.

[4] Ruhmann S., Joly A. G. and Nelson K. A., J. Chem. Phys., 86 (1987) 6563; Ruhmann S., KöHler B., Joly A. G. and Nelson K. A., IEEE J. Quantum Electron., 24 (1988) 470.

[5] Chesnoy J. and Mokhtari A., Phys. Rev. B, 38 (1988) 3566.

[6] Cheng T. K., Brorson S. D., Kazeroonian A. S., Moodera J. S., Dresselhaus G., Dresselmaus M. S. and Ippen E. P., Appl. Phys. Lett., 57 (1990) 1004; Cheng T. K., Vidal S., Zeiger M. J., Dresselhaus G., Dresselhaus M. S. and IpPen E. P., Appl. Phys. Lett., 59 (1991) 1923.

[7] Chwalek J. M., Uher C., Whitaker J. F., Mourou G. A. and Agostinelli J. A., Appl. Phys. Lett., 58 (1991) 980.

[8] Pfeifer T., Kütt W., Kurz H. and Scholz R., Phys. Rev. Lett., 69 (1992) 3248.

[9] KÜtT W., Albrecht W. and KURZ H., IEEE J. Quantum Electron., 28 (1992) 2434.

[10] Cно G. C., KÜTT W. and Kurz H., Phys. Rev. Lett., 65 (1990) 764.

[11] Pfeifer T., Dekorsy T., KÜtT W. and KURz H., Appl. Phys. A, 55 (1992) 482. 
[12] Zeiger H. J., Vidal J., Cheng T. K., Ippen E. P., Dresselhaus G. and Dresselhaus M. S., Phys. Rev. B, 45 (1992) 768.

[13] Yan Y. X., Gamble E. B. and Nelson K. A., J. Chem. Phys., 83 (1985) 5391.

[14] Weiner A. M., Leaird D., Wiederrecht G. P. and Nelson K. A., J. Opt. Soc. Am. B, 8 (1991) 1264.

[15] Spicer W. E., Liliental-Weber Z., Weber E., Newman N., Kendelewicz T., Cao R., McCants C., Mahowald P., Miyano K. and Lindau I., J. Vac. Sci. Technol. B, 6 (1988) 1245.

[16] Sze S. M., Physics of Semiconductor Devices, 2nd edition (Wiley, New York, N.Y.) 1981.

[17] Dekorsy T., Pfeifer T., KütT W. and Kurz H., Phys. Rev. B, 47 (1993) 3842.

[18] Min L. and Miller R. J. D., Appl. Phys. Lett., 56 (1990) 524.

[19] YARIF A., Introduction to Optical Electronics (Holt, Rinehart and Winston, New York, N.Y.) 1971.

[20] Strahmen M., KütT W. and KURz H., in Proceedings of an International Conference on $V M E$-bus in Research, edited by C. EcK (North-Holland, Amsterdam) 1988, p. 69.

[21] Vallée F. and Bogani F., Phys. Rev. B, 43 (1991) 12049.

[22] KüTT W., in Festkörperprobleme/Advances in Solid State Physics, Vol. 32, edited by U. RössLER (Vieweg) 1992.

[23] Williams D. A., Phys. Rev. Lett., 69 (1992) 2551. 